\title{
CRECIMIENTO DE PLÁNTULAS DE DOS ESPECIES DE MEZCAL EN FUNCIÓN DEL TIPO DE SUELO Y NIVEL DE FERTILIZACIÓN
}

\section{SEEDLING GROWTH OF TWO MEZCAL SPECIES AS AFFECTED BY SOIL TYPE AND FERTILIZATION RATE}

\author{
Saúl Martínez-Ramírez ${ }^{1,2 *}$, Antonio Trinidad-Santos ${ }^{1}$, Gilberto Bautista-Sánchez ${ }^{2}$ \\ y E. César Pedro-Santos
}

\begin{abstract}
${ }^{1}$ Colegio de Postgraduados-Campus Montecillo. Km 36.5 Carr. México-Texcoco. 56230, Montecillo, Texcoco, Estado de México. ${ }^{2}$ Universidad Tecnológica de la Mixteca. Km 2.5 Carr. Huajuapan-Acatlima. 69000, Huajuapan de León, Oaxaca, México. Tel. 01(953) 5322933 Ext. 550 y Fax 01 (953) 5320214.

*Autor para correspondencia (saulmr@mixteco.utm.mx, ramar-gable@hotmail.com)
\end{abstract}

\section{RESUMEN}

Los magueyes de la Mixteca oaxaqueña no han sido objeto de fertilización, a pesar de que se emplean para producir mezcal. En este estudio se evaluó en invernadero la influencia de tres suelos (Acatlima, Leptosol; Miltepec, Regosol; y Yodoyuxi, Regosol) y de cuatro dosis de fertilización con 0-0-0, 30-20-15, 60-40-30 y 90-60-45 kg de N-P-K $\mathrm{ha}^{-1}$, en el crecimiento y producción de biomasa de plántulas de dos especies de Agave (A. angustifolia Haw. y A. potatorum Zucc.). Se empleó un arreglo factorial de tratamientos $2 \times 3 \times 4$ bajo un diseño experimental completamente al azar. Se evaluaron 24 tratamientos con cuatro repeticiones y 96 unidades experimentales (plantas). Siete meses después de la germinación se evaluaron las variables: altura de planta (A), diámetro de roseta (D), número de hojas desplegadas (HD) y biomasa seca (BS). Los datos se sometieron a análisis de varianza y pruebas de Tukey $(\alpha=0.05)$. El suelo de Miltepec promovió que la plántulas produjeran 300 y $220 \%$ más BS que los suelos de Acatlima y Yodoyuxi, respectivamente. Las plántulas fertilizadas con 30-20-15 kg de N-P-K ha-1 tuvieron $58.3 \%$ más BS que las plántulas no fertilizadas. No se detectó interacción alguna de importancia en el crecimiento de las dos especies de agave mezcalero. Se concluyó que el suelo de Miltepec y la fertilización con 30-20-15 kg de N-P-K ha ${ }^{-1}$ fueron los factores que más favorecieron el crecimiento y el rendimiento de biomasa de A. angustifolia y A. potatorum.

Palabras clave: Agave angustifolia, A. potatorum, altura, biomasa, diámetro, hojas desplegadas.

\section{SUMMARY}

Effect of fertilization in agaves at the Mixteca region has not been assessed, even though they are utilized for mezcal production. In this study, seedling growth of two species (Agave angustifolia Haw. and A. potatorum Zucc.) cultivated under greenhouse conditions in three soil types (Acatlima, Miltepec and Yodoyuxi soils) combined with four fertilization rates $(0-0-0,30-20-15,60-40-30$ and $90-60-45 \mathrm{~kg}$ of $\mathrm{N}-\mathrm{P}-\mathrm{K} \mathrm{ha}^{-1}$ ), was evaluated. A factorial arrangement $2 \times 3 \times 4$ with 24 treatments and four replicates and 96 experimental units (plants) were distributed in a completely random design. Plant height and diameter, number of unfolded leaves, and dry biomass (BS) were measured 7 months after germination. Analyses of variance and Tukey tests $(\alpha=$ 0.05) were carried out for means comparison. It was found that the soil of Miltepec allowed agave plantlets to generate 300 and $220 \%$ more BS than the Acatlima or Yodoyuxi soils, respectively. Seedlings fertilized with 30-20-15 kg de N-P-K ha-1 yielded 58.3\% more BS than seedlings without fertilization. Interactions between main factors had not any important contribution on agave growth. It is concluded that plantlet growth and yield of $A$. angustifolia and $A$. potatorum were promoted by the soil of Miltepec and by fertilization with 30-20-15 $\mathrm{kg}$ of N-P-K ha-1.

Index words: Agave angustifolia, A. potatorum, biomass, diameter, plant height, unfolded leaves.

\section{INTRODUCCIÓN}

La calidad del suelo y la fertilización son factores que determinan el crecimiento de las plantas. Los magueyes mezcaleros Agave angustifolia y A. potatorum se distribuyen en la cuenca alta del río Mixteco. Acatlima, Miltepec y Yodoyuxi son tres localidades de esta cuenca, en las que ambas especies forman parte de la vegetación forestal (Blanco et al., 2001). En la Mixteca de Oaxaca estos agaves son objeto de recolección para la elaboración de mezcal. Según Sánchez (2005), el mezcal de A. potatorum tiene más demanda y se llega a cotizar hasta $300 \%$ más caro que el obtenido de otros magueyes. Esta diferencia se atribuye a su sabor conferido por la proporción de compuestos aromáticos volátiles (Molina et al., 2007; Vera et al., 2009; Vera-Guzmán et al., 2010).

Para alentar su cultivo se necesita generar tecnología que permita obtener mayores rendimientos de materia prima para mezcal, y también otros bienes y servicios como: alimento, fibra, material para construcción, medicina, combustible, cobijo, ornato y herramientas agrícolas, al igual que lo hacían los pueblos indígenas desde hace miles de años (Sánchez, 1997; García-Mendoza, 2007). Los magueyes también tienen potencial para producir combustible y fijar carbono (Colunga-GarcíaMarín et al., 2007; Borland et al., 2009; Rendón-Salcido et al., 2009; Chambers y Holtum, 2010; García-Moya et al., 2010; Núñez et al., 2010), por lo que cada vez son más importantes como sustitutos de fuentes de combustibles y para mitigar efectos del cambio climático global.

La cantidad de hojas que despliegan los magueyes está 
relacionada con su crecimiento, por lo que dicha cantidad constituye una variable apropiada para evaluar el efecto de la fertilización (Nobel et al., 1988). Así, en A. lechuguilla las dosis que estimularon al despliegue de hojas fueron 100 $\mathrm{kg}$ de $\mathrm{N}$ y $500 \mathrm{~kg}$ de $\mathrm{P} \mathrm{ha}^{-1}$, aplicados por separado, y que también incrementaron la asimilación neta de $\mathrm{CO}_{2}$ en $24 \mathrm{~h}$, mientras que la aplicación de $500 \mathrm{~kg}$ de $\mathrm{K} \mathrm{ha}^{-1} \mathrm{o} 100 \mathrm{~kg}$ de $\mathrm{B}$ ha ${ }^{-1}$ redujo la asimilación neta de $\mathrm{CO}_{2}$ e inhibió el despliegue de hojas (Nobel et al., 1988). Según Nobel et al. (1989),

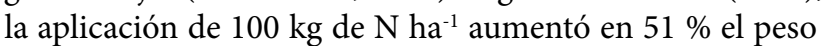
seco de la biomasa aérea en los dos años de evaluación, sin efecto en biomasa seca de raíz, y la aplicación de $500 \mathrm{~kg}$ de $\mathrm{P} \mathrm{ha}^{-1}$ aumentó en $40 \%$ la biomasa seca de la parte aérea de la planta pero disminuyó la de raíz en $37 \%$, de modo que las mejores dosis de N, P, K y B fueron 100, 500, 100 y 100 $\mathrm{kg} \mathrm{ha}^{-1}$, respectivamente.

Por su parte, en A. tequilana Valenzuela y González (1995) determinaron que el rendimiento estimado de la piña (tallo cortado) se duplicó con respecto al tratamiento testigo, cuando las plantas se fertilizaron con $120 \mathrm{~N}-80 \mathrm{P}-60 \mathrm{~K}$, mientras que la aplicación de $120 \mathrm{~N}-120 \mathrm{P}-60 \mathrm{~K}$ generó rendimientos menores. Debido a que en la Mixteca oaxaqueña los suelos son pobres en fertilidad (Blanco et al., 2001) y están limitados por la erosión y clima para la producción agrícola (Alfaro, 2004), en este trabajo se planteó evaluar el efecto de tres suelos de diferente fertilidad y cuatro dosis de fertilización N-P-K, en el crecimiento y producción de biomasa de plántulas de $A$. angustifolia y A. potatorum.

\section{MATERIALES Y MÉTODOS}

El trabajo se llevó a cabo en Montecillo, Texcoco, Estado de México, de julio de 2005 a enero de 2006, con plántulas de A. angustifolia y A. potatorum. Las semillas se recolectaron en Acatlima, Huajuapan de León, Oaxaca, a partir de magueyes silvestres. Los tres suelos a evaluar se adquirieron en sitios donde se distribuyen de manera natural las especies estudiadas: Acatlima, Santiago Miltepec y San Pedro Yodoyuxi. Blanco et al. (2001) determinaron que el tipo de suelo para el primer sitio es Leptosol, y en los otros es Regosol. En cada sitio el suelo se extrajo de un área de 50 x $50 \mathrm{~cm}$ hasta $30 \mathrm{~cm}$ de profundidad donde se distribuyen las raíces. Las características de los suelos (Cuadro 1) se determinaron mediante los procedimientos descritos en la NOM-021-RECNAT-2000 (DOF, 2001).

Las semillas se sembraron en almácigo preparado con mezcla de tierra lama y tierra de monte en proporción 1:1. La tierra lama es un suelo fértil formado por sedimentos de ríos y arroyos (Ortiz y Gutiérrez, 1999). La tierra de monte es un material de origen mineral y orgánico que se acumula en terrenos forestales, según la NOM-027-SEMARNAT-1996 (DOF, 2003). Las plántulas se trasplantaron a suelo $15 \mathrm{~d}$ después de la germinación, cuando apareció la primera hoja laminar. Como macetas se usaron recipientes de plástico de $1.5 \mathrm{~L}$. El suelo se cribó en malla metálica para remover partículas mayores de $4 \mathrm{~mm}$. El volumen e intervalo de riego se determinó por diferencia de peso; antes del trasplante el suelo se llevó a capacidad de campo (CC), posteriormente las macetas se pesaron diariamente hasta que el peso del agua evaporada llegó a $50 \%$ de la CC, lo cual ocurrió a los $4 \mathrm{~d}$.

Se aplicaron cuatro dosis de N-P-K ( $\left.\mathrm{kg} \mathrm{ha}^{-1}\right)$ : baja, 30-2015; media, 60-40-30; alta, 90-60-45; y sin fertilización, 0000-00, como testigo. Como fuentes de nutrientes se empleó urea ( $46 \%$ de $\mathrm{N}$ ), fosfato di-amónico ( $18 \%$ de $\mathrm{N}$ y $46 \%$ de $\mathrm{P}_{2} \mathrm{O}_{5}$ ) y sulfato de potasio ( $50 \%$ de $\mathrm{K}_{2} \mathrm{O}$ y $18 \%$ de S). Los gránulos de fosfato y de sulfato de potasio se trituraron en un mortero de porcelana y luego se mezclaron con agua, junto con la urea. Se hizo una sola aplicación, una semana después del trasplante (15 de julio de 2005).

Los 24 tratamientos resultantes del arreglo factorial completo $2 \times 3 \times 4$, correspondientes a dos especies de maguey, tres procedencias de suelo y cuatro dosis de fertilización, se asignaron a un diseño experimental completamente al azar con cuatro repeticiones, con un total de 96 unidades experimentales (plantas). Siete meses después de la germinación se midió altura de planta (A), diámetro de roseta (D), se contaron hojas desplegadas (HD) y se determinó biomasa seca (BS). A y D se midieron con un vernier (Pretul ${ }^{\circledR}$; Estado de México, México). Primero

Cuadro 1. Características físicas y químicas de los suelos de Acatlima, Miltepec y Yodoyuxi, Oaxaca.

\begin{tabular}{|c|c|c|c|c|c|c|c|c|c|c|}
\hline \multirow{2}{*}{ Sitio } & Textura & MO & $\mathrm{pH}$ & $\mathrm{CE}$ & CIC & $\mathrm{Ca}^{2+}$ & $\mathrm{Mg}^{2+}$ & $\mathrm{Na}^{+}$ & $\mathrm{K}^{+}$ & \multirow{2}{*}{$\begin{array}{c}\mathrm{P} \\
\left(\mathrm{mg} \mathrm{kg}^{-1}\right)\end{array}$} \\
\hline & arena-limo-arcilla (\%) & $(\%)$ & & $\left(\mathrm{dS} \mathrm{m}^{-1}\right)$ & \multicolumn{5}{|c|}{$\left(\mathrm{cmol}_{(+)} \mathrm{kg}^{-1}\right)$} & \\
\hline Acatlima & $61-14-25$ & 1.4 & 7.8 & 1.5 & 20 & 25 & 2.5 & 0.40 & 0.2 & trazas \\
\hline Miltepec & $21-41-38$ & 4.3 & 6.6 & 1.0 & 20 & 25 & 13 & 0.25 & 0.2 & 3.99 \\
\hline Yodoyuxi & $42-25-33$ & 3.9 & 8.2 & 0.4 & 40 & 25 & 5.5 & 0.13 & 0.4 & trazas \\
\hline
\end{tabular}

MO, CE, CIC, $\mathrm{Ca}^{2+}, \mathrm{Mg}^{2+}, \mathrm{Na}^{+}, \mathrm{K}^{+}$y P son: materia orgánica, conductividad eléctrica, capacidad de intercambio catiónico, calcio intercambiable, magnesio intercambiable, sodio intercambiable, potasio intercambiable y fósforo aprovechable, respectivamente. 
se contó HD, y enseguida las plantas se extrajeron de los recipientes, se lavaron las raíces con agua corriente, y las muestras vegetales se secaron en estufa (Riossa ${ }^{\circledR}$, modelo HCFD-82; México) con circulación de aire forzado a 70 ${ }^{\circ} \mathrm{C}$ hasta peso constante, y al final se pesaron en una balanza analítica (Ohaus ${ }^{\circledR}$; New Jersey, USA).

Los datos se sometieron a análisis de varianza y pruebas de comparación múltiple de medias de Tukey $(\alpha=0.05)$, con el paquete estadístico SAS ${ }^{\circledR}$, versión 9.1 (SAS, 2004). Además de la prueba de F, en las fuentes de variación de factores principales y sus interacciones, se calculó su proporción con respecto a la suma de cuadrados de tratamientos, como propusieron Sahagún-Castellanos et al. (2008) para experimentos factoriales.

\section{RESULTADOS Y DISCUSIÓN}

Los análisis de varianza detectaron que el suelo generó diferencias significativas $(P \leq 0.01)$ en las cuatro variables evaluadas en las plántulas de maguey mezcalero: altura de planta (A), diámetro de roseta (D), hojas desplegadas (HD) y biomasa seca (BS); y que la fertilización tuvo efecto en las variables D, HD y BS; en cambio, el factor especie no generó diferencia alguna en las variables (Cuadro 2). En cuanto a las interacciones, la de especie $\mathrm{x}$ suelo no generó diferencias significativas $(\mathrm{P} \leq 0.05)$, la de especie $\mathrm{x}$ fertilización solamente lo tuvo en BS, la de suelo $\mathrm{x}$ fertilización tuvo efecto en D y BS, y la de especie $\mathrm{x}$ suelo $\mathrm{x}$ fertilización generó diferencias significativas $(\mathrm{P} \leq 0.01)$ en $\mathrm{D}$ y en BS. Sin embargo, la contribución de las interacciones en la suma de cuadrados de tratamientos resultó menor a $10 \%$ (Cuadro 3), lo que indica que la combinación de factores no produjo sinergia importante en el crecimiento de $A$. angustifolia y A. potatorum.
Efecto del suelo. Las plantas cultivadas en suelo de Miltepec tuvieron 75.7, 120, 56.6 y $300 \%$ más altura de planta, diámetro de roseta, hojas desplegadas y biomasa seca que las plantas cultivadas en suelo de Acatlima, el cual resultó menos favorable (Cuadro 4). El mejor crecimiento de las plantas de A. angustifolia y A. potatorum cultivados en suelo de Miltepec se atribuye a que en éste la combinación de características físicas y químicas le confirió mejores condiciones para el crecimiento de los magueyes. Entre los factores que pudieron haber influido destacan los contenidos de materia orgánica (MO), de $\mathrm{Mg}^{2+} \mathrm{y}$ de $\mathrm{P}$.

De acuerdo con Ibarra et al. (2007), el contenido de MO en Miltepec resultó muy rico ( $>4 \%$ ), el de Acatlima pobre (1 a $1.9 \%$ ) y el de Yodoyuxi rico (3 a $3.9 \%$ ). Resultados semejantes fueron encontrados por Navarro et al. (2006), ya que los cultivos que evaluaron produjeron más biomasa en el suelo de Nonoalco que en el de Ixayoc, Estado de México, en donde la primera localidad tuvo contenidos medios de MO y de nitrógeno total ( 1.8 y $0.11 \%$ ) y alto en calcio ( 2543 $\mathrm{mg} \mathrm{kg}{ }^{-1}$ ), en tanto que en Ixayoc había contenidos bajos de MO y nitrógeno total ( 0.6 y $0.04 \%$ ) y medio en calcio ( 1150 $\left.\mathrm{mg} \mathrm{kg}{ }^{-1}\right)$, de modo que el suelo de Nonoalco posee mejores características físicas y químicas.

La MO aumenta la fertilidad del suelo, mejora su estructura e incrementa la retención de agua (Havlin et al., 2005); también contribuye a la formación de macro y micro agregados, lo que propicia la continuidad de poros y facilita así la infiltración de agua en todo el perfil (Sustaita et al., 2000). El fósforo está involucrado en múltiples procesos vitales, aunque su función principal es almacenar y transferir energía, en tanto que el $\mathrm{Mg}^{2+}$ es un constituyente de la clorofila y de los ribosomas, a los que estabiliza para que la síntesis de proteínas se lleve a cabo (Havlin et al., 2005).

Cuadro 2. Significancia de F en las variables de crecimiento, generada por los factores especie, suelo, fertilización, y sus interacciones, en plántulas de $A$. angustifolia y A. potatorum de siete meses de edad.

\begin{tabular}{|c|c|c|c|c|c|c|c|}
\hline \multirow{2}{*}{ Variable } & E & $S$ & $\mathrm{~F}$ & $\mathrm{ExS}$ & $\mathrm{ExF}$ & $S \times F$ & $E \times S \times F$ \\
\hline & \multicolumn{7}{|c|}{$\operatorname{Pr}>F$} \\
\hline A & 0.8583 & $<0.01^{\star \star}$ & 0.1309 & 0.3435 & 0.6689 & 0.5007 & 0.4890 \\
\hline $\mathrm{D}$ & 0.1138 & $<0.01^{\star *}$ & $<0.01^{\star \star}$ & 0.1699 & 0.1423 & $<0.01^{\star *}$ & $<0.01^{* *}$ \\
\hline HD & 0.1038 & $<0.01^{\star \star}$ & $<0.01^{\star *}$ & 0.7223 & 0.1469 & 0.5718 & 0.0523 \\
\hline BS & 0.0699 & $<0.01^{\star *}$ & $<0.01^{\star *}$ & 0.2396 & $<0.01^{\star *}$ & $<0.01^{\star \star}$ & $<0.01^{* *}$ \\
\hline
\end{tabular}

${ }^{*}$ Efecto significativo con $\mathrm{P} \leq 0.05, \mathrm{y}^{* *}$ con $\mathrm{P} \leq 0.01$; E, S, F, A, D, HD y BS son: especie, suelo, dosis de fertilización, altura, diámetro, hojas desplegadas y biomasa seca, respectivamente. 
Cuadro 3. Contribución relativa (\%)de las fuentes de variación de los tratamientos aplicados a plántulas de Agave angustifolia y A. potatorum) de siete meses de edad, con respecto a la suma de cuadrados del modelo.

\begin{tabular}{lrrrr}
\hline \multirow{2}{*}{ Fuentes de variación para tratamientos } & \multicolumn{4}{c}{ Contribución de la suma de cuadrados (\%) } \\
\cline { 2 - 5 } & $\mathrm{A}$ & $\mathrm{D}$ & $\mathrm{HD}$ & $\mathrm{BS}$ \\
\hline Especie & 0.0 & 1.0 & 1.6 & 0.9 \\
Suelo & 83.2 & 69.8 & 75.9 & 73.3 \\
Fertilización & 4.8 & 11.1 & 7.9 & 4.9 \\
Especie x suelo & 1.8 & 1.3 & 0.4 & 0.8 \\
Especie x fertilización & 1.3 & 2.1 & 3.3 & 6.4 \\
Suelo x fertilización & 4.4 & 7.1 & 2.9 & 5.7 \\
Especie x suelo x fertilización & 4.5 & 7.7 & 8.0 & 8.1 \\
Suma & 100.0 & 100.0 & 100.0 & 100.0 \\
\hline
\end{tabular}

A, D, HD y BS son: altura, diámetro, hojas desplegadas y biomasa seca, respectivamente.

Cuadro 4. Promedios ( $n=48$, especie; $n=32$, suelo; $n=24$, dosis de fertilización) de las variables de crecimiento medidas en plántulas de A. angustifolia y A. potatorum de siete meses de edad.

\begin{tabular}{|c|c|c|c|c|c|c|c|c|c|}
\hline \multirow[t]{2}{*}{ Variable } & \multicolumn{2}{|c|}{ Especie } & \multicolumn{3}{|c|}{ Suelo } & \multicolumn{4}{|c|}{ Dosis de fertilización N-P-K ${ }^{\dagger}$} \\
\hline & A. ang & A. pot & Aca & Mil & Yod & Sin & baja & media & alta \\
\hline $\mathrm{A}(\mathrm{cm})$ & 4.7 & 4.8 & $3.7 \mathrm{~b}$ & $6.5 \mathrm{a}$ & $4.1 \mathrm{~b}$ & 4.3 & 5.1 & 4.7 & 4.9 \\
\hline $\mathrm{D}(\mathrm{cm})$ & 8.9 & 9.6 & $6.0 \mathrm{c}$ & $13.2 \mathrm{a}$ & $8.6 \mathrm{~b}$ & $7.3 \mathrm{~b}$ & $9.6 \mathrm{a}$ & $10.3 \mathrm{a}$ & $9.9 \mathrm{a}$ \\
\hline HD & 6.3 & 6.7 & $5.3 \mathrm{~b}$ & $8.3 \mathrm{a}$ & $5.9 \mathrm{~b}$ & $5.8 \mathrm{~b}$ & $6.6 \mathrm{ab}$ & $6.9 \mathrm{a}$ & $6.7 \mathrm{a}$ \\
\hline BS (g) & 1.5 & 1.8 & $0.8 \mathrm{~b}$ & $3.2 \mathrm{a}$ & $1.0 \mathrm{~b}$ & $1.2 \mathrm{~b}$ & $1.9 \mathrm{a}$ & $1.8 \mathrm{a}$ & $1.7 \mathrm{a}$ \\
\hline
\end{tabular}

Medias sin letras o con letras iguales en la misma hilera de especie, suelo y dosis de fertilización no son estadísticamente diferentes (Tukey, 0.05). A. ang, A. pot, Aca, Mil, Yod, son: A. angustifolia, A. potatorum, Acatlima, Miltepec y Yodoyuxi, respectivamenge; ${ }^{\dagger}$ sin, baja, media y alta corresponden a las dosis 0-0-0, 30-20-15, 60-40-30, 90-60-45 kg N-P-K ha-1 ${ }^{-1}$ respectivamente; A, D, HD y BS son: altura, diámetro, hojas desplegadas y biomasa seca, respectivamente.

Efecto de la fertilización. Las plántulas fertilizadas con cualquiera de las dosis de N-P-K crecieron más $(\mathrm{P} \leq 0.05)$ que las del testigo, en diámetro de roseta, hojas desplegadas y acumulación de biomasa, pero no en altura de planta (Cuadro 4). La mejor dosis de fertilización para plántulas de maguey fue la de $30 \mathrm{~N}-20 \mathrm{P}-15 \mathrm{~K}$. Al igual que en la mayoría de las plantas cultivadas, la fertilización con NPK resultó benéfica para el crecimiento y producción de biomasa de $A$. angustifolia y $A$. potatorum, lo que coincide con los resultados encontrados por Nobel et al. (1988) en plantas adultas de A. lechuguilla. En este estudio, sin embargo, la dosis óptima para plántulas resultó menor que la encontrada por los autores citados (100 kg de $\mathrm{N} \mathrm{ha}^{-1}$ ) para plantas adultas, lo que puede deberse a diferencias en edades de las plantas y en especies.

La mejor dosis (30-20-15) para plántulas de A. angustifolia y A. potatorum es inferior a la de 40-40-30 que se emplea en plantaciones comerciales de $A$. tequilana en la cuenca hidrográfica "El Jihuite" (Flores-López et al., 2009), y también menor que la dosis de $50 \mathrm{~kg}$ de $\mathrm{N} \mathrm{ha}^{-1}$ recomendada para A. salmiana cultivada de temporal o secano (Medina et al., 2003). Pero es cercana a la dosis de $\mathrm{N}$ que se emplea en plantaciones comerciales de A. angustifolia en la región del mezcal, donde se aplican $50 \mathrm{~g}$ de sulfato de amonio por planta (SAGARPA, 2004), equivalente a $21 \mathrm{~kg} \mathrm{de} \mathrm{N} \mathrm{ha}^{-1}$ con una densidad de 2000 plantas ha ${ }^{-1}$.

A diferencia de las especies C3 de importancia agrícola, A. angustifolia y A potatorum con metabolismo ácido de las crasuláceas (MAC) tienen menor avidez por la fertilización $\mathrm{N}-\mathrm{P}-\mathrm{K}$, lo que sugiere que son más eficientes en el uso de estos nutrimentos, aunque esto no ha sido totalmente esclarecido (Lüttge, 2004). Según Lin (2009), las plantas MAC usan menor cantidad de nitrógeno, lo cual se asocia con el hecho de que muchas plantas MAC habitan en sustratos con deficiencias severas de nutrientes.

Efecto de especie x suelo. Según la prueba de $\mathrm{F}$ en los análisis de varianza, la interacción especie de maguey $\mathrm{x}$ tipo de suelo no generó efectos significativos (Cuadro 2) en el crecimiento de las plántulas, ya que en el mejor de los casos el efecto fue de apenas $1.8 \%$ en la variable altura (Cuadro 3). Sin embargo, la prueba de Tukey $(\alpha=0.05)$ mostró que las plántulas de $A$. potatorum y de $A$. angustifolia que se cultivaron en suelo de Miltepec crecieron más 
en altura, diámetro de roseta, hojas desplegadas y biomasa seca que cuando se cultivaron en suelos de Acatlima o de Yodoyuxi (Cuadro 4). Estas diferencias son atribuibles en gran proporción al factor suelo, más que a la combinación con la especie de maguey. Con base en lo anterior, es claro que la sinergia entre especie de maguey y tipo de suelo fue de escaso valor en promover el crecimiento de las plántulas.

Efecto de especie $x$ dosis de fertilización. Los tratamientos correspondientes a la interacción especie $\mathrm{x}$ fertilización generaron diferencias significativas $(\mathrm{P}<0.01)$ sólo en biomasa seca (Cuadro 2), ya que solamente las plántulas de A. potatorum que se fertilizaron con cualquiera de las tres dosis tuvieron más biomasa seca que las del testigo. Sin embargo, la contribución de esta interacción a la suma de cuadrados de los tratamientos, sólo contribuyó con el $6.4 \%$, lo cual indica que la diferencia que se obtuvo con la prueba de Tukey para la separación de medias (Cuadro 5) es atribuible a la dosis de fertilización más que a la combinación de los factores especie con las dosis de fertilización. De esta manera se concluye que en este caso tampoco existió sinergia importante entre la especie y la fertilización que favorecieran el crecimiento de las plántulas de A. angustifolia y A. potatorum.

Efecto de suelo $\mathrm{x}$ fertilización. Según los análisis de varianza, los tratamientos correspondientes a la combinación suelo $\mathrm{x}$ fertilización generaron diferencias significativas ( $\mathrm{P}$ $<0.01$ ) en las variables diámetro de roseta y en biomasa seca (Cuadro 2). Sin embargo, la contribución de esta interacción en la suma de cuadrados de tratamientos apenas fue de 7.1 y $5.7 \%$, respectivamente(Cuadro 3). Así, la diferencias en las respuestas mostradas en el Cuadro 6, son atribuibles más a la fertilización que a su combinación con el factor suelo, por lo que se concluye que no hubo sinergia entre los tipos de suelo estudiados y las dosis de fertilización aplicadas en favor de un mejor crecimiento de las plántulas de $A$. angustifolia y A. potatorum.

Efecto de especie $\mathbf{x}$ suelo $\mathrm{x}$ fertilización. Al igual que en los tratamientos formados por la combinación de suelo y fertilización, los análisis de varianza indicaron que los tratamientos correspondientes a la interacción de los tres factores, tuvieron efectos significativos $(\mathrm{P}<0.01)$ en diámetro de roseta y en biomasa seca (Cuadro 2). A pesar de esto, la contribución de la triple interacción apenas contribuyó con 7.7 y $8.1 \%$ respectivamente, a la suma de cuadrados (Cuadro 3), por lo que las diferencias que se muestran el Cuadro 7 son más atribuibles al efecto del tipo de suelo y de la fertilización que a la combinación de los tres factores. Por ello se concluye que esta interacción tampoco favoreció significativamente al crecimiento de las plántulas de las dos especies de maguey estudiadas.

Cuadro 5. Promedios ( $\mathrm{n}=16$, especie $\mathrm{x}$ suelo; $\mathrm{y} n=12$, especie $\mathrm{x}$ dosis de fertilización) de las variables de crecimiento medidas en plántulas de A. angustifolia y A. potatorum de siete meses de edad.

\begin{tabular}{|c|c|c|c|c|c|c|c|c|c|c|c|c|c|c|}
\hline \multirow{3}{*}{ Variable } & \multicolumn{6}{|c|}{ Especie x suelo } & \multicolumn{8}{|c|}{ Especie $\mathrm{x}$ dosis de fertilización ${ }^{\dagger}$} \\
\hline & \multicolumn{3}{|c|}{ A. angustifolia } & \multicolumn{3}{|c|}{ A. potatorum } & \multicolumn{4}{|c|}{ A. angustifolia } & \multicolumn{4}{|c|}{ A. potatorum } \\
\hline & Aca & Mil & Yod & Aca & Mil & Yod & Sin & Baja & Media & Alt & Sin & Baja & Media & Alta \\
\hline $\mathrm{A}(\mathrm{cm})$ & $3.9 \mathrm{~b}$ & $6.4 \mathrm{a}$ & $3.9 \mathrm{~b}$ & $3.5 \mathrm{~b}$ & $6.6 \mathrm{a}$ & $4.3 \mathrm{~b}$ & 4.3 & 5.3 & 4.5 & 4.8 & 4.3 & 4.9 & 4.9 & 5.0 \\
\hline $\mathrm{D}(\mathrm{cm})$ & $5.8 \mathrm{c}$ & $12.3 \mathrm{a}$ & $8.7 \mathrm{~b}$ & $6.1 \mathrm{c}$ & $14.1 \mathrm{a}$ & $8.6 \mathrm{~b}$ & $7.3 \mathrm{~b}$ & $9.8 \mathrm{ab}$ & $9.6 \mathrm{ab}$ & $8.9 \mathrm{ab}$ & $7.2 \mathrm{~b}$ & $9.3 \mathrm{ab}$ & $11.0 \mathrm{a}$ & $10.9 \mathrm{~b}$ \\
\hline $\mathrm{HD}$ & $5.2 \mathrm{~b}$ & $7.9 \mathrm{a}$ & $5.8 \mathrm{~b}$ & $5.4 \mathrm{~b}$ & $8.6 \mathrm{a}$ & $6.1 \mathrm{~b}$ & $5.7 \mathrm{~b}$ & $6.8 \mathrm{ab}$ & $6.7 \mathrm{ab}$ & $6.1 \mathrm{ab}$ & $5.9 \mathrm{ab}$ & $6.4 \mathrm{ab}$ & $7.1 \mathrm{ab}$ & $7.3 \mathrm{a}$ \\
\hline BS (g) & $0.8 \mathrm{~b}$ & $3.0 \mathrm{a}$ & $0.8 \mathrm{~b}$ & $0.7 \mathrm{~b}$ & $3.4 \mathrm{a}$ & $1.2 \mathrm{~b}$ & $1.3 \mathrm{bc}$ & $2.2 \mathrm{a}$ & $1.6 \mathrm{abc}$ & $1.1 \mathrm{c}$ & $1.1 \mathrm{c}$ & $1.6 \mathrm{abc}$ & $2.0 \mathrm{ab}$ & $2.3 \mathrm{a}$ \\
\hline
\end{tabular}

Medias sin letras o con letras iguales en la misma hilera de especie $\mathrm{x}$ suelo y especie $\mathrm{x}$ dosis de fertilización no son estadísticamente diferentes (Tukey, 0.05). Aca, Mil y Yod son: Acatlima, Miltepec y Yodoyuxi, respectivamente; ${ }^{\dagger}$ sin, baja, media y alta corresponden a las dosis 0-0-0, 30-20-15, 60-40-30, 90-60-45 kg N-P-K ha ${ }^{-1}$, respectivamente; A, D, HD y BS son: altura, diámetro, hojas desplegadas y biomasa seca, respectivamente.

Cuadro 6. Promedios $(n=8)$ de variables de crecimiento medidas en plántulas de siete meses de A. angustifolia y A. potatorum crecidas en suelo de Acatlima, Miltepec y Yodoyuxi, Oaxaca, y sometidas a cuatro dosis de fertilización ${ }^{\dagger}$.

\begin{tabular}{|c|c|c|c|c|c|c|c|c|c|c|c|c|}
\hline \multirow{2}{*}{ Variable } & \multicolumn{4}{|c|}{ Acatlima } & \multicolumn{4}{|c|}{ Miltepec } & \multicolumn{4}{|c|}{ Yodoyuxi } \\
\hline & Sin & Baja & Media & Alta & Sin & Baja & Media & Alta & Sin & Baja & Media & Alta \\
\hline $\mathrm{A}(\mathrm{cm})$ & $3.7 \mathrm{~d}$ & $3.6 \mathrm{~d}$ & $3.6 \mathrm{~d}$ & $4.0 \mathrm{~d}$ & $6.1 \mathrm{abc}$ & $7.3 \mathrm{a}$ & $6.2 \mathrm{abc}$ & $6.4 \mathrm{ab}$ & $3.2 \mathrm{~d}$ & $4.5 \mathrm{bcd}$ & $4.2 \mathrm{~cd}$ & $4.3 \mathrm{~cd}$ \\
\hline $\mathrm{D}(\mathrm{cm})$ & $5.6 \mathrm{~d}$ & $5.0 \mathrm{~d}$ & $6.9 \mathrm{~cd}$ & $6.5 \mathrm{~cd}$ & $11.2 \mathrm{ab}$ & $14.2 \mathrm{a}$ & $14.6 \mathrm{a}$ & $12.8 \mathrm{ab}$ & $5.0 \mathrm{~d}$ & $9.6 \mathrm{bc}$ & $9.4 \mathrm{bc}$ & $10.5 \mathrm{~b}$ \\
\hline HD & $4.8 \mathrm{~d}$ & $5.0 \mathrm{~d}$ & $5.9 \mathrm{~d}$ & $5.5 \mathrm{~d}$ & $7.9 \mathrm{abc}$ & $8.5 \mathrm{a}$ & $8.4 \mathrm{a}$ & $8.3 \mathrm{ab}$ & $4.8 \mathrm{~d}$ & $6.3 \mathrm{~cd}$ & $6.4 \mathrm{bcd}$ & $6.3 \mathrm{~cd}$ \\
\hline $\mathrm{BS}(\mathrm{g})$ & $0.8 \mathrm{~d}$ & $0.6 \mathrm{~d}$ & $0.9 \mathrm{~d}$ & $0.8 \mathrm{~d}$ & $2.3 \mathrm{bc}$ & $4.0 \mathrm{a}$ & $3.4 \mathrm{ab}$ & $3.0 \mathrm{ab}$ & $0.5 \mathrm{~d}$ & $1.1 \mathrm{~d}$ & $1.2 \mathrm{~d}$ & $1.3 \mathrm{~cd}$ \\
\hline
\end{tabular}

Medias con letras iguales en la misma hilera no son estadísticamente diferentes (Tukey, 0.05 ). ${ }^{\dagger} \sin$, baja, media y alta corresponden a las dosis 0-0-0, 30-20-15, 6040-30, 90-60-45 kg N-P-K ha-1 , respectivamente; A, D, HD y BS son: altura, diámetro, hojas desplegadas y biomasa seca, respectivamente. 
Cuadro 7. Crecimiento de plántulas de A. angustifolia y A. potatorum de siete meses de edad cultivadas en suelos de tres localidades (Acatlima, Miltepec y Yodoyuxi, Oaxaca) y con cuatro dosis de fertilización.

Especie $\mathrm{x}$ suelo $\mathrm{x}$ dosis de fertilización ${ }^{\dagger}$

Variable

Agave angustifolia

\begin{tabular}{|c|c|c|c|c|c|c|c|c|}
\hline & \multicolumn{4}{|c|}{ Acatlima } & \multicolumn{4}{|c|}{ Miltepec } \\
\hline & Sin & Baja & Media & Alta & Sin & Baja & Media & Alta \\
\hline $\mathrm{A}(\mathrm{cm})$ & 4.3 abcdefg & $4.3 \mathrm{abcdefg}$ & $3.2 \mathrm{defg}$ & $3.9 \mathrm{cdefg}$ & 6.0 abcdef & $7.2 \mathrm{ab}$ & $6.2 \mathrm{abcd}$ & $6.2 \mathrm{abcd}$ \\
\hline $\mathrm{D}(\mathrm{cm})$ & $4.7 \mathrm{hi}$ & $5.9 \mathrm{ghi}$ & $5.8 \mathrm{ghi}$ & 6.9 fghi & $12.5 \mathrm{abcdef}$ & 13.1 abcde & $14.2 \mathrm{abcd}$ & 9.4 cdefghi \\
\hline $\mathrm{HD}$ & $4.5 \mathrm{e}$ & $5.5 \mathrm{cde}$ & $5.3 \mathrm{de}$ & $5.5 \mathrm{cde}$ & $8.3 \mathrm{abc}$ & $8.5 \mathrm{ab}$ & $8.3 \mathrm{abc}$ & 6.8 bcde \\
\hline \multirow[t]{3}{*}{ BS (g) } & $0.8 \mathrm{f}$ & $0.8 \mathrm{f}$ & $0.7 \mathrm{f}$ & $0.9 \mathrm{f}$ & $2.7 \mathrm{bcde}$ & $4.6 \mathrm{a}$ & $3.1 \mathrm{abcd}$ & $1.5 \mathrm{def}$ \\
\hline & \multicolumn{4}{|c|}{ Yodoyuxi } & & & & \\
\hline & $\operatorname{Sin}$ & Baja & Media & Alta & & & & \\
\hline $\mathrm{A}(\mathrm{cm})$ & $2.6 \mathrm{~g}$ & 4.5 abcdefg & 4.0 bcdefg & 4.3 abcdefg & & & & \\
\hline $\mathrm{D}(\mathrm{cm})$ & $4.9 \mathrm{ghi}$ & $10.5 \mathrm{abcdefg}$ & 8.8 defghi & $10.6 \mathrm{abcdefg}$ & & & & \\
\hline HD & $4.3 \mathrm{e}$ & $6.3 \mathrm{bcde}$ & 6.5 bcde & $6.0 \mathrm{bcde}$ & & & & \\
\hline BS (g) & $0.3 \mathrm{f}$ & $1.1 \mathrm{ef}$ & $1.0 \mathrm{f}$ & $1.0 \mathrm{f}$ & & & & \\
\hline
\end{tabular}

Agave potatorum

\begin{tabular}{|c|c|c|c|c|c|c|c|c|}
\hline \multirow[b]{3}{*}{$\mathrm{A}(\mathrm{cm})$} & \multicolumn{4}{|c|}{ Acatlima } & \multicolumn{4}{|c|}{ Miltepec } \\
\hline & Sin & Baja & Media & Alta & Sin & Baja & Media & Alta \\
\hline & $3.0 \mathrm{efg}$ & $2.8 \mathrm{gf}$ & 4.1 bcdefg & 4.0 bcdefg & $6.2 \mathrm{abcd}$ & $7.4 \mathrm{a}$ & 6.1 abcde & $6.5 \mathrm{abc}$ \\
\hline $\mathrm{D}(\mathrm{cm})$ & 6.4 ghi & 4. hi & 8.0 efghi & $6.1 \mathrm{ghi}$ & 10.0 bcdefgh & $15.3 \mathrm{ab}$ & $15.1 \mathrm{abc}$ & $16.1 \mathrm{a}$ \\
\hline $\mathrm{HD}$ & $5.0 \mathrm{de}$ & $4.5 \mathrm{e}$ & 6.5 bcde & $5.5 \mathrm{cde}$ & $7.5 \mathrm{abcd}$ & $8.5 \mathrm{ab}$ & $8.5 \mathrm{ab}$ & $9.8 \mathrm{a}$ \\
\hline \multirow[t]{3}{*}{ BS (g) } & $0.8 \mathrm{f}$ & $0.3 \mathrm{f}$ & $1.1 \mathrm{ef}$ & $0.7 \mathrm{f}$ & $1.9 \mathrm{cdef}$ & $3.4 \mathrm{abc}$ & $3.7 \mathrm{ab}$ & $4.4 \mathrm{a}$ \\
\hline & \multicolumn{4}{|c|}{ Yodoyuxi } & & & & \\
\hline & Sin & Baja & Media & Alta & & & & \\
\hline $\mathrm{A}(\mathrm{cm})$ & 3.8 cdefg & $4.5 \mathrm{abcdefg}$ & 4.4 abcdefg & 4.4 abcdefg & & & & \\
\hline $\mathrm{D}(\mathrm{cm})$ & $5.1 \mathrm{ghi}$ & 8.7 defghi & $10.0 \mathrm{bcdefgh}$ & $10.5 \mathrm{abcdefg}$ & & & & \\
\hline HD & $5.3 \mathrm{de}$ & 6.3 bcde & 6.3 bcde & 6.5 bcde & & & & \\
\hline BS (g) & $0.7 \mathrm{f}$ & $1.2 \mathrm{ef}$ & $1.3 \mathrm{ef}$ & $1.7 \mathrm{def}$ & & & & \\
\hline
\end{tabular}

Medias con letras iguales en la misma hilera no son estadísticamente diferentes (Tukey, 0.05 ). ${ }^{\dagger}$ sin, baja, media y alta corresponden a las dosis 0-0-0, 30-20-15, 60 40-30, 90-60-45 kg N-P-K ha-1 ${ }^{-1}$ respectivamente; A, D, HD y BS son: altura, diámetro, hojas desplegadas y biomasa seca, respectivamente.

\section{CONCLUSIONES}

Las plántulas de A. angustifolia y A. potatorum mostraron igual crecimiento en altura, diámetro, hojas desplegadas y biomasa. El suelo de Miltepec resultó mejor para el crecimiento de A. angustifolia y A. potatorum, que los suelos de Acatlima y Yodoyuxi. La fertilización con 30-20-15 kg de $\mathrm{N}-\mathrm{P}-\mathrm{K} \mathrm{ha}^{-1}$ favoreció el crecimiento de ambas especies. No se detectó sinergismo por la combinación de los tres factores estudiados, especie, dosis de fertilización y tipo de suelo.

\section{BIBLIOGRAFÍA}

Alfaro S G (2004) Suelos. In: Biodiversidad de Oaxaca. García-Mendoza A J, M J Ordóñez, M Briones-Salas (eds). Instituto de Biología, UNAM-Fondo Oaxaqueño para la Conservación de la Naturaleza-World Wildlife Fundation. México. pp:55-65.

Blanco A A, S Martínez, O Sánchez, A Rubio, C Cisneros, E C Pedro, R Morales, F Sustaita (2001) Aplicación de un Modelo de Balances Hídricos en la Cuenca Alta del Río Mixteco (Oaxaca). Determinación del Binomio Infiltración/escurrimiento con Vistas a la Reconstrucción de sus Ecosistemas Forestales. Universidad Tecnológica de la Mixteca, Huajuapan de León, Oaxaca. México. $251 \mathrm{p}$.

Borland A M, H Griffiths, J Hartwell, J A C Smith (2009) Exploiting the potential of plants with crassulacean acid metabolism for 
bioenergy production on marginal lands. J. Exp. Bot. 60:28792896.

Chambers D, J A M Holtum (2010) Feasibility of Agave as a Feedstock for Biofuel Production in Australia. Rural Industries Research and Development Corporation. Australian Government. $\mathrm{Pu}-$ blication No 10/104. 74 p. Disponible en: http://eprints.jcu.edu. $\mathrm{au} / 15163 / 1 / 2010$ _Chambers_ and_Holtum.pdf (diciembre 2013).

Colunga-GarcíaMarín P, A Larqué, L E Eguiarte, D Zizumbo-Villarreal (2007) El futuro de lo ancestral. In: En lo Ancestral hay Futuro: del tequila, los mezcales y otros agaves. M P Colunga-García A Larqué, L E Eguiarte, D Zizumbo-Villarreal (eds). Centro de Investigación Científica de Yucatán, A. C. Mérida, Yucatán. México. pp:395-402.

DOF, Diario Oficial de la Federación (2001) Norma Oficial Mexicana NOM-021-RECNAT-2000 que establece las especificaciones de fertilidad, salinidad y clasificación de suelos. Estudios, muestreo y análisis. Disponible en: http://www.profepa.gob. $\mathrm{mx} /$ innovaportal/file/3335/1/nom-021-semarnat-2000.pdf (diciembre 2013).

DOF, Diario Oficial de la Federación (2003) Norma Oficial Mexicana NOM-027-SEMARNAT-1996 que establece los procedimientos, criterios y especificaciones para realizar el aprovechamiento, transporte y almacenamiento de tierra de monte. Disponible en: http://www.profepa.gob.mx/innovaportal/file/341/1/ nom-027-semarnat-1996.pdf (diciembre 2013).

Flores-López H E, R Carrillo-González, N Francisco-Nicolás, C Hidalgo-Moreno, J A Ruiz-Corral, A A Castañeda-Villanueva, $R$ Velazco-Nuño (2009) Aportes de nitrógeno y fósforo de tres sistemas agrícolas de la cuenca hidrográfica "El Jihuite", en Jalisco, México. Agrociencia 23:659-669.

García-Mendoza A J (2007) Los agaves de México. Ciencias 87:14-23.

García-Moya E, A Romero-Manzanares, P S Nobel (2010) Highlights for Agave productivity. Global Change Biol. Bioen. 3:4-14.

Havlin J L, J D Beaton, S L Tisdale, W L Nelson (2005) Soil Fertility and Fertilizers. An Introduction to Nutrient Management. 7th ed. Pearson Prentice Hall. Upper Saddle River, New Jersey, U. S. A. 515 p.

Ibarra C D, J A Ruiz, J G Flores, D R González (2007) Distribución espacial del contenido de materia orgánica de los suelos agrícolas de Zapopan, Jalisco. Terra Latinoam. 25:187-194.

Lin Ch C (2009) The effects of environmental factors in the induction of crassulacean acid metabolism (CAM) expression in facultative CAM plants. JULS 3:64-66.

Lüttge U (2004) Ecophysiology of crassulacean acid metabolism (CAM). Ann. Bot. 93:629-652.

Medina G, A Rumayor, B Cabañas, M Luna, J A Ruiz, C Gallegos, J Madero, R Gutiérrez, S Rubio, A G Bravo (2003) Potencial productivo de especies agrícolas en el estado de Zacatecas. Instituto Nacional de Investigaciones Forestales, Agrícolas y Pecuarias. Libro Técnico No. 2. Centro de Investigación Regional
Norte Centro. Campo Experimental Zacatecas. Calera, Zacatecas, México. 157 p.

Molina G J A, J E Botello, A Estrada, J L Navarrete, H Jiménez, M Cárdenas, R Rico (2007) Compuestos volátiles en el mezcal. Rev. Mex. Ing. Quím. 6:41-50.

Navarro G H, M A Pérez, F Castillo (2006) Evaluación de cinco especies vegetales como cultivos de cobertura en valles altos de México. Rev. Fitotec. Mex. 30: 151-157.

Nobel P S, E Quero, H Linares (1988) Differential growth responses of agaves to nitrogen, phosphorus, potassium and boron applications. J. Plant Nutr. 11:1683-1700.

Nobel P S, E Quero, H Linares (1989) Root versus shoot biomass: responses to water, nitrogen, and phosphorus applications for Agave lechuguilla. Bot. Gaz. 150:411-416.

Núñez H M, L F Rodríguez, M Khanna (2010) Agave for tequila and biofuels: an economic assessment and potential opportunities. Global Change Biol. Bioen. 3:43-57.

Ortiz S C A, Ma. Del C Gutiérrez C (1999) Evaluación taxonómica de sistemas locales de clasificación de tierras. Terra 17:277-286.

Rendón-Salcido L A, P Colunga-GarcíaMarín, L F Barahona-Pérez, E Pimienta-Barrios, A Magdub-Méndez, A Larqué-Saavedra (2009) Sugars and alcohol by products from Henequén (Agave fourcroydes) as influenced by plant age and climate. Rev. Fitotec. Mex. 32:39-44.

SAGARPA, Secretaría de Agricultura Ganadería Desarrollo Rural Pesca y Alimentación (2004). Diagnóstico de la Cadena Productiva del Sistema Producto Maguey-mezcal. SAGARPA-Delegación Oaxaca, Oaxaca, México. 213 p.

Sahagún-Castellanos J, A Martínez-Garza, J E Rodríguez-Pérez (2008) Problemas y métodos comunes de análisis de experimentos factoriales. Rev. Chapingo Ser. Hortic. 14:213-222.

Sánchez L A (1997) El mezcal en la historia de México: de Oaxaca, su mezcal. Bebidas Mex. 6:7-10

Sánchez L A (2005) Oaxaca, Tierra de Maguey y Mezcal. 2a. ed. Instituto Tecnológico de Oaxaca. Oaxaca. México. 235 p.

SAS Institute (2004) SAS 9.1 SQL Procedure User's Guide. Cary, NC, USA.

Sustaita R F, V Ordaz, C A Ortiz, F de León (2000) Cambios en las propiedades físicas de dos suelos de una región semiárida debidos al uso agrícola. Agrociencia 34:379-386.

Valenzuela Z G, D R González (1995) Fertilización del agave tequilero (Agave tequilana Weber) en la región de Tequila, Jalisco, México. Ensayo de una metodología para analizar crecimiento en cultivos multianuales mediante una técnica no destructiva. Terra 13:81-95.

Vera G A M, P A Santiago, M G López (2009) Compuestos volátiles aromáticos generados durante la elaboración de mezcal de Agave angustifolia y Agave potatorum. Rev. Fitotec. Mex. 32:273-279.

Vera-Guzmán A M, R I Guzmán-Gerónimo, M G López (2010) Major and minor compounds in a Mexican spirit, young mescal coming from two Agave species. Czech J. Food Sci. 28:127-132. 